\title{
TITLE:
}

\section{$<$ Note $>$ Hand Clapping by a Chimpanzee in the Nimba Mountains, Guinea, West Africa}

$\operatorname{AUTHOR}(S)$ :

Koops, Kathelijne; Matsuzawa, Tetsuro

CITATION:

Koops, Kathelijne ... [et al]. <Note> Hand Clapping by a Chimpanzee in the Nimba Mountains, Guinea, West Africa. Pan Africa News 2006, 13(2): $19-21$

\section{ISSUE DATE:}

2006-12

URL:

http://hdl.handle.net/2433/143467

RIGHT:

Copyright (C) Pan Africa News. 


\section{Kathelijne Koops ${ }^{1}$ and Tetsuro Matsuzawa² \\ IL everhulme Centre for Human Evolutionary Studies and Dept. of Biological Anthropology, \\ University of Cambridge \\ 2Primate Research Institute, Kyoto University}

\section{INTRODUCTION}

We report the first observation of hand clapping by a wild chimpanzee (Pan troglodytes verus) in the Nimba Mountains in Guinea, West Africa. Hand dapping has previously been reported only for captive chimpanzees (Pan troglodytes). There, chimpanzees have been seen to clap hands in the context of potential food availability to attract the attention of humans $\mathrm{s}^{1,2}$ and as a gesture during play3. Also, bonobos (Pan paniscus) in the San Diego Zoo hand- and foot-clapped during grooming ${ }^{4}$, presenting a unique local tradition that has been transmitted to new individuals introduced into the group 5,6 . For wild great apes, hand clapping has been seen only in one female mountain gorilla (Gorilla gorilla beringer) in the Virunga Mountains, Rwanda7, and in several female and young western lowland gorillas (Gorilla gorilla gorilla) in southwestern Central African Republic ${ }^{8}$. Most cases in which hand clapping was seen in western lowland gorillas were when the gorillas were nervous about the presence of the human observers ${ }^{8}$.

Our chimpanzee study site is in a part of the Nimba Mountains near the village of Seringbara $\left(7^{\circ} 37^{\prime} 50.0^{\prime \prime} \mathrm{N}, 8^{\circ} 27^{\prime} 44.7^{\prime \prime} \mathrm{W}\right)$ in southeastern Guinea. The Seringbara study area covers about $25 \mathrm{~km}^{2}$ and is $6 \mathrm{~km}$ southeast of Bossou, where a community of 12-23 dhimpanzees has been studied since 19769, 10. Occasional surveys in the Nimba area have been ongoing since 1992. However, no constant research presence in the Nimba Mountains around Seringbara existed before the present study (begun in August 2003) and the chimpanzees remain largely unhabituated.

\section{OBSERVATIONS}

At $10.30 \mathrm{~h}$ on 12 May, 2004, KK and field assistants found an adult female chimpanzee eating ripe fruit in a Parkia bicolor tree. When the chimpanzee saw the researchers, she barked and screamed and shook branches. Two other 
chimpanzees ( 1 adult and 1 juvenile) quickly moved away. The adult female continued to scream and to bark intermittently at the observers and threw several branches. After about 30 minutes, she calmed down and continued to eat Parkia bicolor fruit and to groom herself for several minutes.

She then moved to a nearby Canarium schweinfurthii tree and peered at the researchers before branch-shaking, 'waa-barking' and hand clapping. She continued intermittently to feed on Canarium schweinfurthii fruit and on young leaves, self-groom and bark/scream at the observers in combination with hand-to-foot and hand-to-hand clapping. During the total period of observation (131 minutes) she showed two hand-clapping bouts ( 3 claps; 4 claps) and three hand-to-foot clapping bouts (1 clap; 2 claps; 1 clap) before departing at $12.41 \mathrm{~h}$. All hand-to-hand and hand-to-foot clapping bouts were associated with a particular type of 'waa-bark'. The observations were captured on film (see video clip. Video dip of this behaviour is available at the following URL. http://mahale. web.infoseek.co.jp/PAN/koops_video/)

\section{DISCUSSION}

Our preliminary report describes a previously unknown communication gesture in wild chimpanzees in Nimba. However, in the early stages of habituation with irregular observations of the chimpanzees, it is not yet clear whether hand-dapping and hand-to-foot clapping is idiosyncratic, habitual or customary in the Nimba chimpanzees.

The context of the behaviour seems to resemble that in which hand-dapping was recorded in wild western lowland gorillas8. Hand-to-hand and hand-to-foot clapping appear to be used in threat or display, similar to branch-shaking. The combination of vocalizations and clapping may alert other chimpanzees in the area to the presence of potential danger (e.g. humans). Also, the behaviour may reflect a combination of fear and frustration, in response to the presence of human observers. Only further research on the chimpanzees in the Nimba Mountains can provide information about the generality of this behavioural pattern and its form and function.

\section{ACKNOWLEDGMENTS}

The research was financially supported by MEXT grants (12002009 and 16002001) and J SPS core-to-core program HOPE and 21COE (A14) to T. Matsuzawa and by a grant from the Lucie Burgers Foundation for Comparative Behavioural Research, Arnhem, the Netherlands, to K. Koops. In Guinea, we thank the Ministère de l'Enseignement Supérieur et de la Recherche Scientifique, Direction Nationale de la Recherche Scientifique, and Institut de Recherche Environnementale de Bossou for granting us the permission to carry out this study. We also thank T. Humle and the Seringbara guides for invaluable assistance in the field, and W.C. McGrew for helpful comments on the manuscript.

\section{REFERENCES}

1. Hostetter AB, Cantero M, Hopkins WD 2001. Differential use of vocal and gestural communication by chimpanzees (Pan troglodytes) in response to the attentional status of a human (Homo sapiens). J Comp Psychol 115: 337-343.

2. Fletcher AW 2006. Clapping in chimpanzees: evidence of exclusive hand preference in a spontaneous, bimanual gesture. Am J Primatol 68: 1081-1088.

3. Tomasello M, Call J , Nagell K, Olguin R, Carpenter $M$ 1994. The learning and use of gestural signals by young chimpanzees: a trans-generational study. Primates 35: 137-154.

4. Ingmanson EJ 1987. Clapping behavior: non-verbal communication during grooming in a group of captive pygmy chimpanzees. AmJ Primatol 72: 214 (Abstract).

5. de Waal FBM 1989. Behavioral contrasts between bonobo and chimpanzee. In: Understanding Chimpanzees, Heltne P, Marquardt L (eds), Harvard Univ. Press, Cambridge, MA, pp. 154-175.

6. de Waal FBM 1994. The chimpanzee's adaptive potential: a comparison of social life under captive and wild conditions. In: Chimpanzee Cultures, Wrangham RW, McGrew WC, de Waal FBM, Heltne $P$ (eds), Harvard Univ. Press, Cambridge, MA, pp. 243-260.

7. Fossey D 1983. Gorillas in the Mist. Houghton Mifflin Company, Boston.

8. Fay J M 1989. Hand-dapping in western lowland gorillas (Gorilla gorilla gorilla). Mammalia 53: 457-458.

9. Sugiyama $Y$ 1981. Observation on the population dynamics and behavior of wild chimpanzees at Bossou, Guinea, between 1976-1983. Primates 22: 
435-444.

10. Sugiyama $Y$ 1984. Population dynamics of wild chimpanzees at Bossou, Guinea, between 19761983. Primates 25: 391-400. 